\title{
Biogenic Nano-Synthesis; towards the Efficient Production of the Biocompatible Gold Nanoparticles
}

\author{
Gajanan Ghodake, ${ }^{a}$ Chi-Yong Eom, ${ }^{\dagger, a}$ Si Wouk Kim, ${ }^{\dagger}$ and EonSeon Jin* \\ Department of Life Science, College of Natural Sciences, Hanyang University, Seoul 133-791, Korea \\ *E-mail: esjin@hanyang.ac.kr \\ ${ }^{\dagger}$ Environment and Metabolomics Research Team, Seoul Center, Korea Basic Science Institute, Seoul 136-713, Korea \\ ${ }^{\ddagger}$ Department of Environmental Engineering, Chosun University, 501-759, Korea \\ Received April 22, 2010, Accepted August 16, 2010
}

\begin{abstract}
We present a rapid biogenic method for the production of nanoscale gold particles using pear extract. The formation and stability of pear-derived gold nanoparticles (Pear-AuNPs) were monitored by ultraviolet-visible spectroscopy. Their morphology, elemental composition and crystalline phase were determined by transmission electron microscopy, energydispersive X-ray spectroscopy and selected area electron diffraction. The average core size of crystalline Pear-AuNPs was in the range of $10 \pm 5 \mathrm{~nm}$ and the observed morphology was spherical. The X-ray photoelectron spectrum showed a strong peak for the pure 'Au' phase. The circular dichroism spectrum indicated the natural capping ability of the pear extract, which generated peptide-gold nanoparticles. These nanoparticles were stable in aqueous solution for two months. A cell viability assay of Pear-AuNPs showed biocompatibility with human embryonic kidney 293 cells. Accordingly, this eco-friendly process for the bio-mimetic production of Pear-AuNPs is nontoxic in nature; consequently, it will find potential application in nano-biotechnology.
\end{abstract}

Key Words: Biogenic, AuNPs, Cell viability assay, Nontoxic green product

\section{Introduction}

Chemical synthesis is a popular approach to the production of metal nanoparticles; however, it requires the use of toxic and aggressive chemicals as reducing and/or capping agents. ${ }^{1,2}$ Green nanoscience/nanotechnology is the use of green chemistry principles to design nanoscale products, enhance nano-material production and importantly, to evaluate the toxicity of nanomaterials. ${ }^{3}$ Green chemistry should be integrated into nanotechnologies at the source especially when nano-materials are to be used in medical applications, which include imaging, drug delivery, disinfection and tissue repair. ${ }^{4}$ Research on gold nanoparticles is important due to their unique and tunable surface plasmon resonance (SPR), ${ }^{5}$ and their potential applications in biomedical science. ${ }^{6}$ There is a great demand for the application of biogenic synthesis to the production of gold nanoparticles to avoid toxicity problems; when metal nanoparticles are intended for application to the human body, toxic chemicals should be excluded entirely from the process. ${ }^{7}$

Biological approaches using microorganisms and plants or plant extracts for metal nanoparticle synthesis have been suggested as valuable alternatives to chemical methods. ${ }^{8-10}$ The use of plants for the preparation of nanoparticles could be more advantageous, ${ }^{11}$ because it does not require elaborate processes such as intracellular synthesis and multiple purification steps or the maintenance of microbial cell cultures. ${ }^{7}$ Several plants and their parts have been successfully used for the extracellular synthesis of metal nanoparticles. ${ }^{7}$ Pear fruits are known to be rich in phytochemicals, antioxidants, vitamins, proteins, organic acids and amino acids. ${ }^{12,13}$ Recently we reported, the synthesis

${ }^{\mathrm{a}}$ Both authors equally contributed to this work. of gold nano-plates using pear extract.

Herein, we report on a single step and completely green biosynthesis of gold nanoparticles using pear extract. The nanoparticles were monitored by ultraviolet-visible (UV-vis) spectroscopy and characterized by high-resolution transmission electron microscopy (HR-TEM). X-ray photoelectron spectroscopy (XPS) and energy dispersive spectroscopy (EDS) analysis were employed to determine the phase and to confirm the purity of the gold nanoparticles. The capping of pear proteins and their conformation were confirmed by circular dichroism (CD) spectral analysis. To examine the biocompatibility of gold nanoparticles, a cell viability assay was performed with human embryonic kidney (HEK) 293 cells.

\section{Experimental Section}

Materials and preparation of pear extract. Chloroauric acid $\left(\mathrm{HAuCl}_{4}\right)$ was purchased from Sigma-Aldrich Korea. Fresh pears were purchased from the local market. In typical preparation of pear extract, $100 \mathrm{~g}$ of peeled yellow pear slices were ground in a blender, filtered through mesh and centrifuged twice at $11,000 \times g$ for $15 \mathrm{~min}$ at $4{ }^{\circ} \mathrm{C}$ to remove cell-free debris. The resulting supernatant was then filtered through a $0.2 \mu \mathrm{m}$ filter and employed for the synthesis of gold nanoparticles. Doubledistilled water was used to dilute the aqueous chloroauric acid stock solution and the original pear extract during synthesis and to disperse the resulting gold nanoparticles.

Pear extract-mediated biosynthesis of gold nanoparticles. In this study pear extract is used to obtain phytochemically-derived reducing agents for the generation and stabilization of gold nanoparticles. The nanoparticles were examined for their con- 
sistency in SPR properties and reduction rate by varying the concentration of the pear extract. The same plasmon resonance band was observed at $540 \mathrm{~nm}$ at various concentrations (data not shown), indicating uniformity in the formation of gold nanoparticles. In a typical experiment, dark conditions and a preincubation at $90{ }^{\circ} \mathrm{C}$ were applied separately to a $2 \mathrm{mM} \mathrm{HAuCl}_{4}$ aqueous solution and the pear extract to achieve temperature equilibrium and the final total reaction mixture volume was $20 \mathrm{~mL}$. Biosynthesis of gold nanoparticles (Pear-AuNPs) was begun by adding pear extract at $50 \%(\mathrm{v} / \mathrm{v})$ with a final concentration of $\mathrm{HAuCl}_{4}$ of $2 \mathrm{mM}$. The formation of nanoparticles was monitored by UV-vis spectroscopy. The mixture was centrifuged at $11,000 \times g$ for $15 \mathrm{~min}$ at $4{ }^{\circ} \mathrm{C}$. The process of centrifugation and redispersion was repeated three times to remove unbound pear phytochemicals. Rapidly produced PearAuNPs within 20 min were collected and purified by repeated centrifugation as described above, and used to determine physicochemical and biocompatible properties.

Characterization of gold nanoparticles. A $0.5 \mathrm{~mL}$ aliquot of reaction mixture was periodically harvested, diluted with the same volume of water and monitored with a UV-vis spectrophotometer (Shimadzu, UV-1650 PC) using a $10 \mathrm{~mm}$ optical path-length quartz cuvette at a resolution of $1 \mathrm{~nm}$ between 400 and $700 \mathrm{~nm}$. Samples for high-resolution transmission electron microscopy (HR-TEM) analysis were prepared on lacey carboncoated copper films. The drop-cast films were allowed to dry prior to measurement on a TEM (FEI Tecnai F20 G' $)$. Elemental analysis was performed using energy dispersive spectroscopy (EDS). X-ray photoelectron (XPS) with a Specs Phoibos 150 analyzer was used to investigate the surface atomic composition and chemical states of the gold nanoparticles. Circular dichroism (CD) spectral analysis of Pear-AuNPs dispersed in water was employed to study peptide capping using a $10 \mathrm{~mm}$ quartz cuvette. After keeping the dispersion at $25^{\circ} \mathrm{C}$ for $5 \mathrm{~min}$, spectra were collected from $250 \mathrm{~nm}$ to $190 \mathrm{~nm}$ using a Jasco 810 spectrophotometer. Scanning was performed at $20 \mathrm{~nm} / \mathrm{min}$ using a response of eight sec, and the average of three scans was recorded. The conformation of peptides on Pear-AuNPs were determined and confirmed by repeated scanning at different concentrations in an aqueous suspension.

In vitro stability of gold nanoparticles. The in vitro stability of Pear-AuNPs was determined by observing the constancy of the SPR band when nanoparticle solutions were combined with water, $\mathrm{NaCl}(10 \%)$ and Dulbecco's Modified Eagle Medium (DMEM), pH 7.4. In brief, $0.5 \mathrm{~mL}$ of gold nanoparticles was added to $0.2 \mathrm{~mL}$ of water, $\mathrm{NaCl}$ or DMEM. Stability with respect to the SPR band was determined by recording the UV-vis absorbance.

Cell culture and viability test. HEK 293 cell cultures were maintained in DMEM. For cell toxicity tests, $2 \times 10^{4}$ cells at the exponential growth phase were seeded in each well of 24-well plates and incubated for $24 \mathrm{~h}$ in a $\mathrm{CO}_{2}$ incubator with $5 \% \mathrm{CO}_{2}$ at $37{ }^{\circ} \mathrm{C}$. A series of dilutions containing 10, 20, 40, 80 and $150 \mu \mathrm{M}$ of gold nanoparticles were added to the growth medium. Cell viability was measured at $24,48,72$, and $96 \mathrm{~h}$ postincubation and calculated with an automated cell counter (Countess, Invitrogen). Viable cells exhibiting normal growth were considered to be $100 \%$ viable.

\section{Results and Discussion}

Pear extract-mediated biosynthesis of gold nanoparticles. We initiated studies to address the possible application of pear extract for synthesis of gold nanoparticles. Pear extract is a reservoir of phytochemicals including organic acids, amino acids, peptides and proteins. ${ }^{12,13}$ In addition, the presence of saccharides in the extract provides synergistic reducing power for the rapid transformation of chloroaurate ions into gold nanoparticles. Simple mixing of pear extract (50\% [v/v]) with $\mathrm{HAuCl}_{4}(2 \mathrm{mM})$ at $90{ }^{\circ} \mathrm{C}$ promptly initiated the appearance of a purple-red color, indicating the formation of gold nanoparticles. This color is generated by the excitation of surface plasmon vibrations in the nanoparticles. ${ }^{7,14}$ Detailed UV-vis spectrophotometric analysis of purple-colored solutions confirmed the presence of the characteristic SPR band (Fig. 1). Pear extract thus performed well as a bioresource for initiating the reduction of $\mathrm{HAuCl}_{4}$, resulting into rapid production of gold nanoparticles. Recently we reported, that proteins and/or peptides from pear could be attributed as reducing agents, however their conformation at alkaline $\mathrm{pH}$ and at room-temperature play role in the formation of nuclei for the plate morphologies, because of slow reduction of gold ions and more time for the secondary growth. ${ }^{15}$ The rapid reduction of ions facilitated by the pear phytochemicals and the high temperature (i.e., high free energy) provided is important factors in the rapid formation of nuclei and control of secondary growth. In earlier reports, the involvement and exploitation of biomolecules and the manipulation of reaction conditions using physical and chemical means have been put forward for the synthesis of metal nanoparticles; however the exact mechanisms have yet to be well explored. ${ }^{7,10,11,16-19}$

Green principles were applied to exploit greener nano-synthesis with the intention of producing risk-free nanoscale gold particles. This study aimed to produce gold nanoparticles under nontoxic conditions, reduce the complexity of current methodologies and enhance their efficiency, characterize the properties of the resulting nano-materials and propose safe biomedical applications. The competence of the pear extract to facilitate the functional reduction of gold ions under aqueous condi-

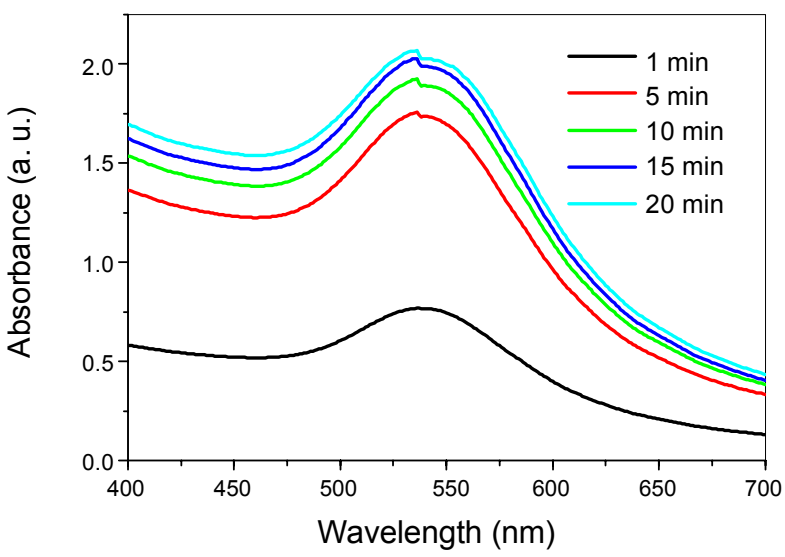

Figure 1. Absorption spectra obtained after $1 \mathrm{~min}$, with or without dilution with the same volume of water, of pear extract and $\mathrm{HAuCl}_{4}$ incubated for $5,10,15$ or $20 \mathrm{~min}$. 


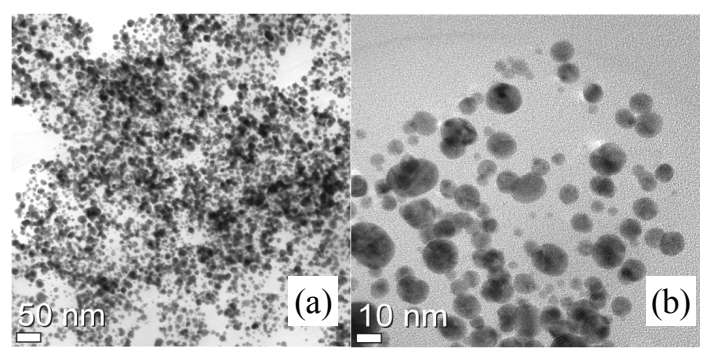

Figure 2. HR-TEM images of gold nanoparticles obtained at $20 \mathrm{~min}$.

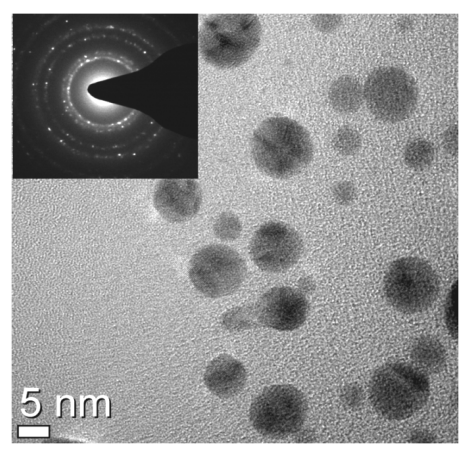

Figure 3. HR-TEM images and corresponding SAED pattern of gold nanoparticles showing their crystal nature.

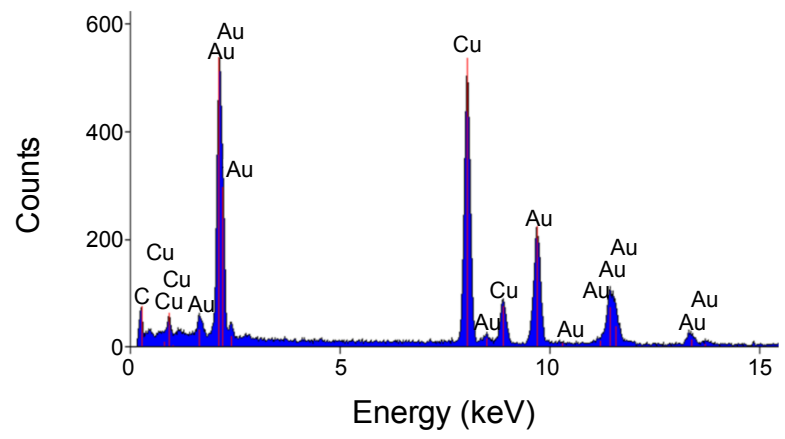

Figure 4. Composition of gold nanoparticles determined by EDS.

tions satisfies the fundamental principles of green chemistry as shown previously. ${ }^{17,18,20,21}$ It has been reported that the synthesis of gold nanoparticles using geranium extract required more time to initiate and was complete in $48 \mathrm{~h}^{22}$ Biosynthesis of silver and gold nanoparticles using bacteria, ${ }^{23-26}$ and fungi, ${ }^{27}$ has been reported, and the time required for completion of the reaction ranged from 24 to $120 \mathrm{~h}$. Intracellular synthesis, prolonged synthesis, multiple purification steps and the maintenance of cell cultures are the drawbacks of microbial procedures. ${ }^{7}$ A simple, efficient, green approach to the production of biocompatible gold nanoparticles using coffee, tea and soybeans has also been described. ${ }^{17,18}$ In addition, Cinnamomum camphora leaf extract has been identified as an effcient reducing and stabilizing agent for the production of gold as well as silver nanoparticles. ${ }^{28}$ The extracellular synthesis of highly stable Ag and Au nanoparticles has also been achieved using Emblica officinalis fruit extract. ${ }^{29}$ Recently, the biosynthesis (a)

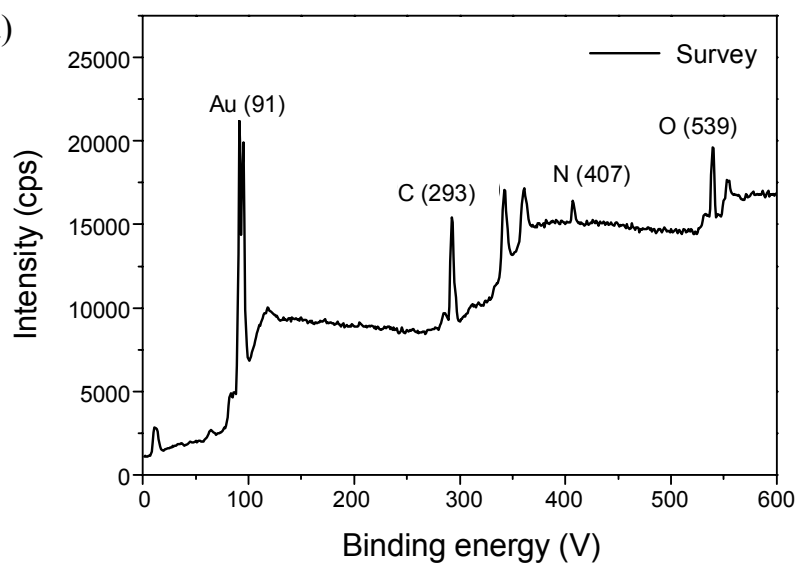

(b)

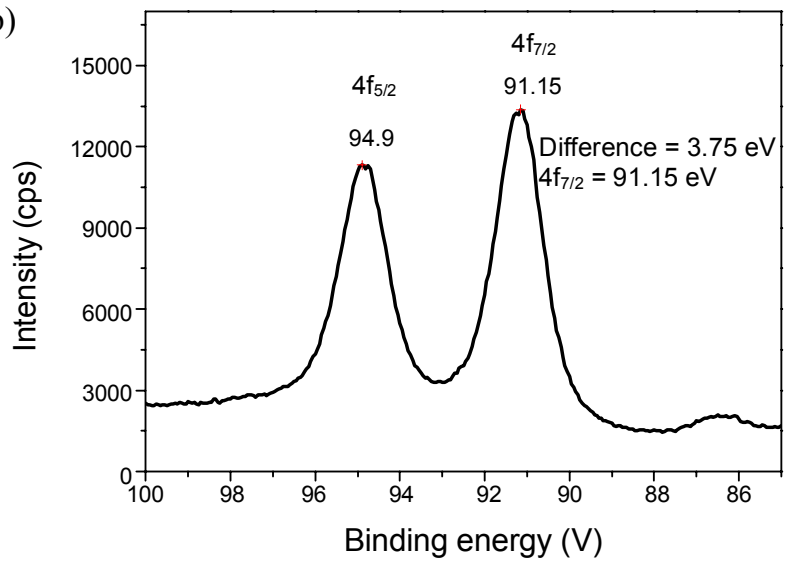

Figure 5. (a) XPS survey spectra of gold nanoparticle thin films, and (b) XPS Au (4/f) spectra of gold nanoparticles.

of biocompatible gold nanoparticles using essential nutritious phytochemicals as well as their possible application in molecular imaging and therapy have been reported. ${ }^{17,18,28,30}$

Characterization of gold nanoparticles. To illustrate the size, morphology, solubility, purity, surface chemistry (capping), physical (e.g., crystal structure) and optical properties of the gold nanoparticles produced herein, UV-vis spectroscopy, HR- TEM, XPS, EDS and CD spectral analysis were performed. Representative TEM images revealed that the nanoparticles were spherical in shape and that the average size of core Pear- AuNPs was $10 \pm 5 \mathrm{~nm}$ (Fig. 2). Most of the particles showed interparticle interactions (Fig. 2), which may have been due to the peripheral complexation of capped biomolecules. The ring-like diffraction pattern indicated that the particles were crystalline in nature. This finding was reflected in the approximately circular nature of the selected area electron diffraction (SAED) spots (Fig. 3). Compositional analysis by EDS illustrated the purity of the gold, with spectra showing a strong Au signal along with a copper signal and weak carbon peaks arising from the carboncoated copper grid (Fig. 4). XPS analysis was performed to determine the composition and surface concentration of gold in nanoparticle thin films. XPS survey spectra revealed a very strong gold signal, confirming the purity of the Pear-AuNPs (Fig. 5a). Two separate peaks due to $\mathrm{Au}\left(4 \mathrm{f}_{7 / 2}\right)$ and $\mathrm{Au}\left(4 \mathrm{f}_{5 / 2}\right)$ transitions located around 91.1 and $94.9 \mathrm{eV}$, respectively, were 


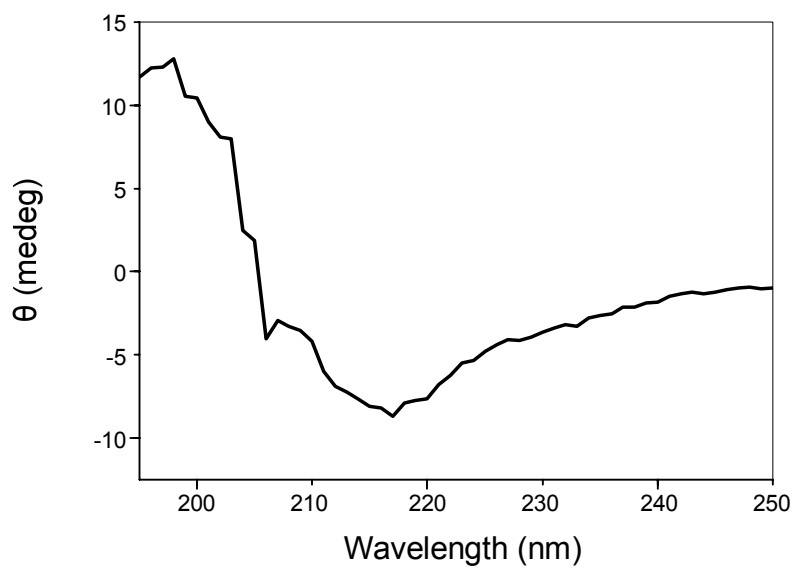

Figure 6. CD spectra of the gold nanoparticle obtained at $20 \mathrm{~min}$.

observed (Fig. 5b). These peaks were allocated to the spin-orbit splitting component of the $\mathrm{Au}\left(4 \mathrm{f}_{7 / 2}\right)$ level in metallic gold, while the binding energies of 84.9 and 88.1 for the Au (4f) peaks corresponded to $\mathrm{Au}^{+}$and $\mathrm{Au}^{3+}$ chemical states, respectively. ${ }^{15,31}$ Therefore, the purity of the gold nanoparticles as determined by EDS and XPS was in good agreement.

The existence and conformation of healthy peptide capping of rapidly produced Pear-AuNPs were identified by CD spectral analysis. The spectrum obtained for Pear-AuNPs dispersed in water showed a negative broad maximum at $220 \mathrm{~nm}$, indicating the existence of stable right-handed $\alpha$-helical structures (Fig. 6). The conformation of peptides or proteins on the PearAuNPs was confirmed by repeated scanning of different concentrations of gold nanoparticles in aqueous suspension. The presence of peptide capping indicated that nanoparticle synthesis was rapid and the capping was natural. The significant degree of peptide coating provides a means of conjugating fluorescent dyes or organic compounds in the preparation of organicinorganic hybrid complexes. Peptide-coated gold nanoparticles have been used in this manner as spacers to conjugate fluorescent dyes. ${ }^{32}$

In vitro stability of gold nanoparticles. An important concern regarding the biomedical application of nanoparticles is their stability in aqueous environments over reasonable periods of time. Therefore, the in vitro stability of Pear-AuNPs was assessed. The absorbance in aqueous mixtures changed by approximately 3 - $5 \mathrm{~nm}$ under all experimental conditions upon combining nanoparticle solutions with water, $\mathrm{NaCl}$ or DMEM. PearAuNPs were unaffected by water and $\mathrm{NaCl}(10 \%)$, whereas in the case of DMEM, both the medium absorbance as well as the SPR band were affected (data not shown). Pear-AuNPs were stable for two month in water, while they were stable for less than two months in $10 \% \mathrm{NaCl}$ (data not shown). It has been shown that a mixture of soybean phytochemicals used to produce gold nanoparticles provided the capping function, ${ }^{17}$ which shielded the nanoparticles against aggregation. The results of this previous report clearly coincide with those of our CD spectral analysis of Pear-AuNPs.

Cell viability of gold nanoparticles. The key parameters in evaluating the biocompatibility of gold nanoparticles are cytotoxicity and cell viability. To this end, we examined the effect

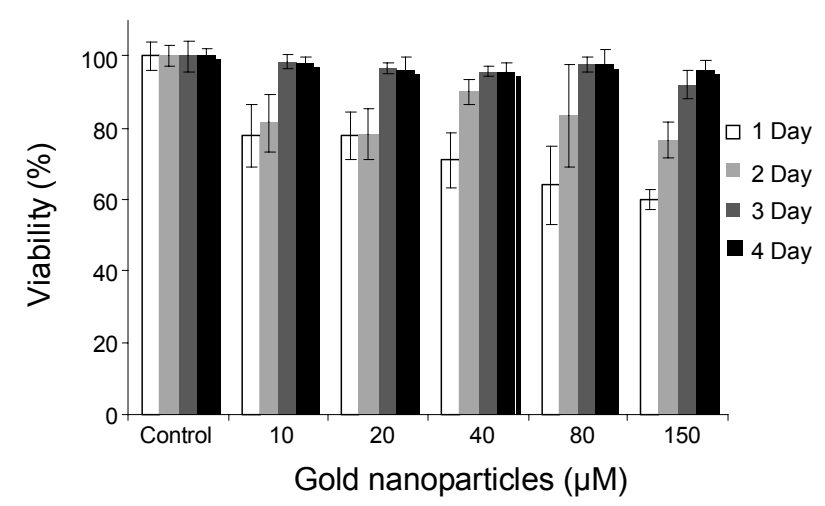

Figure 7. Viability of HEK 293 cells treated with 10, 20, 40, 80 or $150 \mu \mathrm{M}$ gold nanoparticles and measured after incubation for $24 \mathrm{~h}$, $48 \mathrm{~h}, 72 \mathrm{~h}$ and $96 \mathrm{~h}$.

of Pear-AuNPs on cell viability by subjecting HEK 293 cells to concentrations of $10,20,40,80$ and $150 \mu \mathrm{M}$ nanoparticles for $24,48,72$ and $92 \mathrm{~h}$. The cells showed more than 85 to $90 \%$ viability following longer exposures to concentrations of up to $150 \mu \mathrm{M}$ (Fig. 7). As the incubation time increased, biocompatibility increased, reaching $95-99 \%$ in all studied concentrations, indicates the adaptation of the HEK 293 cells to the Pear-AuNP environment. Therefore, the cell viability assay indicated that Pear-AuNPs are nontoxic. The bio-compatibility of AuNPs was increased gradually at all concentrations by time, because the regulation of cell metabolism to new environment and their healthy acceptance as incubation increases. Here there are two possibilities about interaction and metabolism of AuNPs, one is adsorption source to delay in growth, next is metabolism or internalization results viability of cell and healthy growth. Here we believe that, AuNPs prepared by this method are not chronically toxic to the cell growth or for their viability. Nowadays, production of metal nanoparticles under green conditions is a growing concern about toxicity of nanoparticles. $3,18,33-37$

The capability of pear phytochemicals to efficiently reduce chloroaurate ions to biocompatible gold nanoparticles has now been demonstrated. This single-step green method uses pear extract for both the production and subsequent nontoxic biomimetic coating of gold nanoparticles. This approach could find continuing application in diagnostics and therapeutics as previously reported. ${ }^{4,17,28,36}$

\section{Conclusions}

In summary, we demonstrated a competent biosynthetic route for the production of gold nanoparticles using pear extract and $\mathrm{HAuCl}_{4}$ in an aqueous medium. This method is attractive because biocompatible gold nanoparticles can be rapidly produced and stabilized under safe conditions. This nonpolluting biogenic process will be helpful to minimize and/or eliminate chemical interventions and reduce the complexity of metal nanoparticles preparation methodologies. Capping with pear proteins and the nontoxic nature of this "green" product make it a promising candidate for continuing application in the fields of biomedicine and nanotechnology. In addition, this green 
process could be extended to the production of nanoparticles using other noble metals such as silver and platinum.

Acknowledgments. This work was supported by the Pioneer Research Program for Converging Technology of the Ministry of Education, Science and Technology, Republic of Korea (Grant No. M1071118001-08M1118-00110, 2008) and Research fund of HYU (HY-2010-HYURS).

\section{References}

1. Gole, A.; Murphy, C. J. Chem. Mater. 2004, 16, 3633-3640.

2. Meltzer, S.; Resch, R.; Koel, B. E.; Thompson, M. E.; Madhukar, A.; Requicha, A.; Will, P. Langmuir 2001, 17, 1713-1718.

3. Hutchison, J. E. ACS Nano 2008, 2, 395-402.

4. Albrecht, M. A.; Evans, C. W.; Raston, C. L. Green Chem. 2006, $8,417-432$

5. El-Sayed, M. A. Acc. Chem. Res. 2001, 34, 257-264.

6. Huang, X. H.; Jain, P. K.; El-Sayed, I. H.; El-Sayed, M. A. Nanomedicine 2006, 2, 681-693.

7. Kumar, V.; Yadav, S. K. J. Chem. Technol. Biotechnol. 2009, 84, $151-157$

8. Sastry, M.; Ahmad, A.; Khan, M. I.; Kumar, R. In Microbial Nanoparticle Production; Niemeyer, C. M., Mirkin, C. A., Eds.; WileyVCH: Weinheim, Germany, 2004; pp 126-135.

9. Bhattacharya, D.; Gupta, R. Crit. Rev. Biotechno. 2005, 25, 199204.

10. Mohanpuria, P.; Rana, N. K.; Yadav, S. K. J. Nanopart. Res. 2008, 10, 507-517.

11. U.S. Department of Agriculture, Agricultural Research Service. USDA National Nutrient Database for Standard Reference, Release 21. Available from: http://www.nal.usda.gov/fnic/foodcomp/ search. (2008).

12. Chen, J.; Wang, Z.; Wub, J.; Wang, Q.; Hu, X. Food Chem. 2007, 104, 268-275.

13. Mulvaney, P. Langmuir 1996, 12, 788-800.

14. Ghodake, G. S.; Deshpande, N. G.; Lee Y. P.; Jin, E. S. Colloids Surf. B. 2010, 75, 584-589.

15. Ogale, S. B.; Ahmad, A.; Pasricha, R.; Dhas, V. V.; Syed, A. Appl. Phys. Lett. 2006, 89, 263105-263108.
16. Shukla, R.; Nune, S. K.; Chanda, N.; Katti, K.; Mekapothula, S.; Kulkarni, R. R.; Welshons, W. V.; Kannan, R.; Katti, K. V. Small 2008, 4, 1425-1436.

17. Nune, S. K.; Chanda, N.; Shukla, R.; Katti, K.; Kulkarni, R. R.; Thilakavathy, S.; Mekapothula, S.; Kannan, R.; Katti, K. V. J. Mater. Chem. 2009, 19, 2912-2920.

18. Song, J. Y.; Jang, H. K.; Kim, B. S. Process Biochem. 2009, 44, 1133-1138.

19. Poliakoff, M.; Licence, P. Nature 2007, 450, 810-812.

20. Tang, S.; Bourne, R.; Smith. R.; Poliakoff, M. Green Chem. 2008, 10, 268-269.

21. Shankar, S. S.; Rai, A.; Ahmad, A.; Sastry, M. Chem. Mater. 2005, $17,566-572$.

22. Joerger, R.; Klaus, T.; Granqvist, C. G. Adv. Mater. 2000, 12, 407409.

23. Klaus, T.; Joerger, R.; Olsson, E.; Granqvist, C. G. Trends Biotechnol. 2001, 19, 15-20.

24. Nair, B.; Pradeep, T. Cryst. Gro. Des. 2002, 4, 295-298.

25. Ahmad, A.; Senapati, S.; Khan, M.; Kumar, R.; Ramani, R.; Srinivas, V.; Sastry, M. Nanotechnology 2003, 14, 824-828.

26. Mukherjee, P.; Ahmad, A.; Mandal, D.; Senapati, S.; Shankar, S. R.; Khan, M. I.; Parishcha, R.; Ajay, P. V.; Alam, M.; Kumar, R.; Sastry, M. Nano Lett. 2001, 1, 515-519.

27. Huang, J.; Li, Q.; Sun, D.; Lu, Y.; Su, Y.; Yang, X.; Wang, H.; Wang, Y.; Shao, W.; He, N.; Hong, J.; Chen, C. Nanotechnology 2007, 18, 105104-105114.

28. Ankamwar, B.; Damle, C.; Absar, A.; Muraly S. J. Nanosci. Nanotechnol. 2005, 10, 1665-1671.

29. Jain, K. K. Clinical Chem. 2007, 53, 2002-2009.

30. Bulushev, D. A.; Yuranov, I.; Suvorova, E. I.; Buffat, P. A.; KiwiMinsker, L. J. Cat. 2004, 224, 8-17.

31. Santra, S.; Dutta, D.; Walter, G. A.; Moudgil, B. M. Technol. Cancer Res. Treat. 2005, 4, 593-602.

32. Zhang, G.; Keita, B.; Dolbecq, A.; Mialane, P.; Secheresse, F.; Miserque, F.; Nadjo, L. Chem. Mater. 2007, 19, 5821-5823.

33. Gill, S.; Lobenberg, R.; Ku, T.; Azarmi, S.; Roa, W.; Prenner, E. J. J. Biomed. Nanotechnol. 2007, 3, 107-119.

34. Jin, Y. H.; Kannan, S.; Wu, M.; Zhao, J. X. Chem. Res. Toxicol. 2007, 20, 1126-1133.

35. Dahl, J. A.; Maddux, B. L.; Hutchison, J. E. Chem. Rev. 2007, 107, 2228-2269.

36. Lewinski, N.; Colvin, V.; Drezek, R. Small 2008, 4, 26-49. 